\title{
La emergencia de la didáctica de las ciencias sociales: icampo en consolidación o en disgregación?
}

\section{Social Sciences' Didactics Emergence: Desintegrating or Consolidating Field?}

\author{
Sandra Patricia Rodríguez Ávila* \\ Wilson Armando Acosta Jiménez**
}

Resumen

En este artículo se presentan las elaboraciones conceptuales y metodológicas desde las cuales se desarrolla la investigación sobre el estado del arte en la enseñanza y el aprendizaje de las ciencias sociales entre 1990 y 2000; además, se identifica y se conceptualiza el conjunto de enunciados que caracterizan el campo del saber estudiado y sus distintas relaciones; $y$, finalmente, se analizan los procedimientos de control discursivo que configuran la didáctica de las ciencias sociales.

\section{Palabras claves:}

Enseñanza de las ciencias sociales, didáctica de las ciencias sociales, enseñanza, didáctica, discurso educativo, discurso pedagógico.

\section{Abstract}

This article presents the theoretical and methodological elaborations, from which the research about the State of the Arts in the Teaching and Learning of Social Sciences is developed (19902000); this paper also identifies and conceptualizes the set of statements which characterize the studied knowledge area and its different relations; and, finally, it analyzes the procedures of discursive control that build Social Sciences Didactics.

Keywords:

Social Sciences teaching, Social Sciences Didactics, teaching, didactics, educational discourse, pedagogic discourse.

Artículo recibido el 28 de febrero de 2007 y aprobado el 30 de abril de 2007

* Profesora e investigadora del Departamento de Ciencias Sociales de la Universidad Pedagógica Nacional, e investigadora asociada de la Corporación Sociedad Colombiana de Pedagogía.srodriguez@pedagogica. edu.co.

** Profesor del Departamento de Ciencias Sociales de la Universidad Pedagógica Nacional. wilaracostaj@ gmail.com. 


\section{Introducción}

El saber sobre la enseñanza y el aprendizaje de las ciencias sociales en ámbitos escolares se caracteriza por una serie de enunciados que han emergido por efecto del control, selección y redistribución del discurso que, según Michel Foucault (1969), se lleva a cabo en toda sociedad, mediante procedimientos externos que lo delimitan y procedimientos internos que definen sus dinámicas propias. Para hacer visibles estos enunciados y establecer sus relaciones y tensiones, hemos realizado un estado del arte ${ }^{1}$ sobre la producción académica en enseñanza y aprendizaje de las ciencias sociales en los países de habla hispana, que circula en Colombia desde 19902. En este artículo presentamos las elaboraciones conceptuales y metodológicas desde las cuales hicimos el estado del arte, el conjunto de enunciados que caracterizan el campo de saber estudiado y sus distintas relaciones, y planteamos algunas tesis para el debate sobre los procedimientos internos y externos de control discursivo que configuran la didáctica de las ciencias sociales como campo emergente.

1 La importancia de esta modalidad de investigación documental se ha incrementado desde la década de los años ochenta, cuando aparecieron los primeros trabajos derivados de la iniciativa de algunos organismos multilaterales Unesco, Unicef y OEI-, con el fin de hacer un balance bibliográfico sobre la investigación desarrollada en América Latina, en diversos aspectos relacionados con las ciencias sociales -situación de la niñez; la juventud y las mujeres; condiciones socioeconómicas de las poblaciones vulnerables; situación de los desplazados y las victimas de los conflictos; cobertura en salud y educación y calidad de la educación-. Estos estados del arte iniciales sirvieron para establecer el conocimiento acumulado sobre una problemática específica, evaluar la gestión de estos organismos y definir líneas estratégicas de intervención política. Balances de esta naturaleza empezaron a aparecer a escala nacional en los años noventa, con el propósito de identificar el saber producido en distintas líneas disciplinares y temáticas para evaluar la gestión en investigación de instituciones del ámbito nacional-Colciencias- o local-Idep-, o para proponer investigaciones que determinaran algunas líneas temáticas de los trabajos realizados en la enseñanza de las ciencias sociales, sin el propósito de caracterizar dicho campo, sólo con miras a referenciar la producción bibliográfica.

2 Este artículo forma parte de los resultados de la investigación "Estado del arte en la enseñanza y el aprendizaje de las ciencias sociales: producción académica en los países de habla hispana. 1990-2000, que avaló y financió el Ciup-UPN entre 2004 y 2006.

\section{Elaboraciones conceptuales y metodológicas}

En este apartado presentamos algunas elaboraciones sobre el "estado del arte" como modalidad de investigación documental y teórica, con el objeto de contribuir al debate sobre las diferencias conceptuales entre información, conocimiento y campos de saber, y exponemos los criterios de localización, selección y registro de la información, a partir de los cuales se diseñó la base de datos que sirvió de soporte documental para el análisis del campo académico sobre la enseñaza y el aprendizaje de las ciencias sociales.

\section{La investigación documental: información, conocimiento y estados del arte}

La producción, en cualquier ámbito académico, alcanza grandes proporciones en las condiciones actuales. El amplio volumen documental de las bibliotecas, la reestructuración y optimización de los centros de documentación de instituciones especializadas, y la circulación en internet de información de muy diversa naturaleza sobre un mismo objeto de análisis, hacen cada vez más compleja la posibilidad de precisar las dimensiones, características, tensiones, relaciones y dinámicas de un campo de saber específico. En razón de estas circunstancias, se requiere urgentemente caracterizar la producción académica en los campos de saber en los cuales trabajamos, para abordar, analizar y problematizar de modo permanente sus contribuciones en el ámbito educativo. Consideramos pertinente iniciar esta caracterización precisando las diferencias conceptuales entre información y conocimiento, al igual que la manera como los estados del arte contribuyen, mediante un conjunto de información -base de datos-, a construir una base de conocimiento sobre un tema, un problema de análisis, una tendencia teórica o un campo de saber.

Con respecto a los conceptos de información y conocimiento, encontramos que se ha venido naturalizando una cierta simetría conceptual entre información y conocimiento, sobre todo en el marco de las reformas educativas, desde las cuales la dotación tecnológica, el diseño de planes curri- 
culares para incorporar el uso del computador y la adecuación de portales de internet para aumentar la conectividad de docentes y estudiantes, se proponen en función de ampliar las posibilidades de producción del conocimiento tecnológico, pero se evalúan desde la difusión y uso de las TIC (Cepal y Unesco, 1992). En tal sentido, en el discurso "institucional sobre la educación, el concepto de información es utilizado tanto para designar a los productos de las tecnologías teleinformáticas como para dar cuenta de los procesos cognoscitivos involucrados en la creación, circulación y difusión de estas nuevas tecnologías" (Aprea, 2006).

Esta situación viene generando un conjunto de usuarios pasivos -interactuados, en términos de Aprea (2006)-, que tienen un acceso mínimo a opciones estandarizadas de información, por efecto de una imposibilidad cultural de selección y procesamiento de datos relevantes, lo cual aumenta la inequidad y produce un predominio de la información -que circula ampliamente- sobre el conocimiento -sistematizado y acumulado-, afectando tanto los sujetos de discurso -en este caso docentes, investigadores y estudiantes de los niveles universitarios y escolares-, como las formas y dinámicas de los campos de saber y su uso social:

La información, y no el dinero, es la mercancía por excelencia de las redes globales. Pero esta mercancía, en vez de producir una cosificación de las relaciones humanas, lo que hace es volverlas hacia dentro, interiorizándolas en el sentido de la subjetividad. Como han dicho algunos autores, vivimos en la era del narcisismo de la conciencia. La información, en vez de ser un instrumento de la racionalización weberiana del mundo, lo desobjetiviza y vuelve interpretable de mil maneras diferentes. Paradójicamente, la sociedad transparente hacia la cual nos encaminamos será más opaca, barroca y misteriosa que todas las anteriores $(. .$.$) pues llegará el momento en que$ su complejidad habrá superado nuestro limitado radio de comprensión (Bruner, 1999, 20-21).

El efecto del predominio de la información sobre el conocimiento hace que las particularidades conceptuales de este último se pierdan frente a las definiciones del primero. Por tanto, resulta fundamental diferenciar la información como cuerpo de datos sobre un problema social, producidos mediante un ejercicio de investigación o reflexión que se emplea en la determinación de políticas de gestión e intervención social, los cuales se ponen en circulación en las redes de internet y el conocimiento como conjunto de conceptos que operan sobre una realidad objetivada, en este caso de naturaleza educativa, en función de producir tesis en un proceso investigativo.

En una sociedad en la cual hay un desplazamiento cada vez menos evidente del conocimiento por la información, es decir más naturalizado, enraizado e irreflexivo, la formulación y desarrollo de estados del arte permite definir -en la espesura informativa- el rumbo que están tomando los campos de saber, de distintos niveles de formalización -ciencias, disciplinas, ámbitos interdisciplinares o transdisciplinares, estudios temáticos y saberes no sistematizados-. En tal sentido, un estado del arte es una modalidad de investigación sobre el conocimiento producido, acumulado y circulante en distintos formatos bibliográficos, los que se localizan, registran y delimitan en una base documental, sobre la cual se realiza un proceso de clasificación -en un nivel descriptivo-y categorización -en un nivel conceptual-, que en su confluencia permite analizar las dimensiones, relaciones y posiciones estratégicas que caracterizan y constituyen un campo de saber.

\section{Criterios de delimitación en la búsqueda y localización de la información}

$\mathrm{Al}$ abordar la enseñanza y el aprendizaje de las ciencias sociales en el ámbito escolar, el análisis queda circunscrito al desarrollo que han tenido las áreas curriculares en la educación formal; por tanto, se restringió la búsqueda a la producción académica, en la cual las ciencias sociales escolares se abordan desde los siguientes aspectos:

- Caracterización y análisis de la situación de la enseñanza de las ciencias sociales, mediante el estudio de las prácticas de enseñanza; el estado del currículo y su relación con la normatividad oficial; el estado de los procesos de aprendizaje 
escolar en el área mediante su evaluación y sus resultados de rendimiento y desempeño; los textos y manuales escolares que se emplean para la enseñanza y la formación de los docentes del área y su situación profesional.

- Formulación de propuestas pedagógicas y didácticas derivadas de problemáticas identificadas en situaciones escolares genéricas o específicas, que tienen que ver con el aprendizaje, la enseñanza, las disciplinas escolares o el currículo.

- Análisis sobre la conformación de las disciplinas escolares, a partir de las condiciones históricas de aparición de la institución escolar, de la institucionalización y profesionalización de las disciplinas sociales y de las múltiples relaciones que se pueden establecer entre las áreas curriculares y la normatividad oficial, la política educativa y sus procesos de reforma.

- Formulación de propuestas para la formación de docentes en ciencias sociales derivadas de problemáticas identificadas en situaciones escolares genéricas o específicas, vinculadas al ejercicio profesional docente.

No incluimos trabajos relacionados con problemas asociados a la enseñanza de las ciencias sociales, como convivencia y conflicto en la escuela, experiencias de democracia escolar, ciudadanía y educación y formulación y desarrollo de cátedras específicas (afrocolombiana, derechos humanos, multiculturalidad, ciudad educadora), pues no abordan problemas referentes a las disciplinas escolares en los tres ámbitos desde los cuales se delimitó la búsqueda: aprendizaje, enseñanza, disciplinas escolares y currículo, sino que responden a exigencias educativas que desbordan el análisis que se pueda realizar a partir de este estado del arte.

Este tipo de delimitación nos arrojó una muestra significativa de la información circulante sobre este tema, no representativa en términos estadísticos; en este caso, nos hemos centrado en los documentos con soporte físico, más que en los que solamente tienen versión electrónica, debido a las dificultades de su acopio y a que muchas de las publicaciones de este tipo han aparecido sobre todo después de 2002, fuera del período establecido, y en muchos casos son nuevas versiones de documentos publicados con anterioridad en formato impreso.

Para iniciar el proceso de búsqueda de la información pertinente, se diseñó una ficha bibliográfica con los datos básicos de los documentos que correspondían a los siguientes descriptores de búsqueda: ciencias sociales-enseñanza; historia-enseñanza; geografía-enseñanza; ciencias sociales-aprendizaje; historia-aprendizaje; geografía-aprendizaje; didáctica de la historia; didáctica de la geografía y didáctica de las ciencias sociales. Esta primera exploración se llevó a cabo en la Biblioteca Luis Ángel Arango33, en la Biblioteca de la Universidad Pedagógica Nacional ${ }^{4}$ y en la Biblioteca de la Universidad Javeriana 5 .

Con respecto a los sitios de internet encontramos portales especializados como histodidáctica. Enseñanza de la historia/didáctica de las ciencias sociales (http://www.histodidactica.es), y enlaces especializados a grupos de investigación en el área6:

- Grupo de Investigación Dighes (Didáctica de la Geografía e Historia en la Educación Secundaria).

3 En el catálogo de la Biblioteca Luis Ángel Arango, en el campo de palabra clave en título, se filtró la indagación por el período establecido para el estado del arte (1990-2000); se encontraron 20 registros sobre enseñanza de las ciencias sociales; 20 sobre didáctica de las ciencias sociales; 16 sobre enseñanza de la geografía; 7 sobre didáctica de la geografía; 63 sobre enseñanza de la historia y 19 sobre didáctica de la historia; 4 en aprendizaje de las ciencias sociales; 10 en aprendizaje de la historia y 2 en aprendizaje de la geografía. Se empleó como campo para la búsqueda el de palabra clave en el título, para descartar los textos escolares, guías, manuales, cartillas didácticas y materiales educativos. Encontramos un total de 161 registros para el período propuesto.

4 En el catálogo de la Biblioteca de la Universidad Pedagógica Nacional se ubicaron 16 trabajos de grado entre 1990 y 2000, relacionados con la enseñanza y el aprendizaje de las ciencias sociales -propuestas pedagógicas en geografía e historia-; 6 registros de la colección general sobre la enseñanza de las ciencias sociales, que incluyen libros y revistas especializadas; también se ubicaron 5 por didáctica de las ciencias sociales; 5 por aprendizaje de las ciencias sociales; 2 por aprendizaje de la historia; 6 por aprendizaje de la geografía; y, por último, 11 registros por didáctica de la historia y 3 por didáctica de la geografía. Ubicamos un total de 54 registros.

5 Al hacer la búsqueda por el descriptor enseñanza de las ciencias sociales, se relacionan 79 registros -trabajos de grado de la especialización en enseñanza de la historia-; 4 por didáctica de las ciencias sociales -libros y artículos-; 3 por didáctica de la historia. Por los demás descriptores de búsqueda no encontramos registros. El total de documentos ubicados fue 86 .

6 Esta información no se puede cuantificar debido a las condiciones en las cuales circula, pues los documentos que se publican se están actualizando permanentemente. 
- Consejo Superior del Sistema Educativo (Generalitat de Catalunya).

- Departamento de Didáctica de las Ciencias Sociales (Universidad de Barcelona).

- Instituto de Ciencias de la Educación (ICE, Universidad de Barcelona); Organización de Estados Iberoamericanos para la Educación, la Ciencia y la Cultura (OEI).

- Credi (Centro de recursos documentales e Informáticos).

- Asociación Universitaria de Profesorado de Didáctica de las Ciencias Sociales.

- AEHG (Asociación Española de Profesorado de Historia y Geografía).

Igualmente ubicamos centros virtuales de recursos bibliográficos, como la base de datos de la Organización de Estados Iberoamericanos. Credi: Centro de Recursos Documentales e Informáticos, varios de los documentos indexados en los catálogos de consulta de las bibliotecas visitadas, fundamentalmente los publicados por editoriales comerciales. De la base de datos del Instituto para la Investigación Educativa y el Desarrollo Pedagógico-Idep, realizamos el registro del fondo de Informes de Investigación e Innovación y encontramos 29 registros. Por último, en la Hemeroteca Virtual Dialnet,7 que integra diversos recursos de búsqueda de información y de alertas informativas a sus usuarios, hallamos información de los índices de revistas de habla hispana en diversos temas y algunas publicaciones periódicas especializadas en educación, entre las cuales están referenciadas cinco revistas en didáctica de las ciencias sociales8.

7 Esta hemeroteca virtual se inició en 2001 en la Universidad de La Rioja (España) y se fortaleció a partir de 2003, al pasar a ser un proyecto cooperativo en el cual participan varias bibliotecas universitarias.

8 Revista Con-ciencia social: anuario de didáctica de la geografía, la historia y las ciencias sociales de la Federación Icaria de Grupos de Innovación Didáctica de Geografía, Historia y otras Ciencias Sociales (Fedicaria), editada por Diada, con una frecuencia anual desde 1997, y consta de siete números; Revista Didáctica de las ciencias experimentales y sociales del Departamento de Didáctica de las Ciencias Experimentales y Sociales de la Universidad de Valencia, con una frecuencia anual desde 1989, consta de 18 números; revista Didáctica Geográfica de la Asociación de Geógrafos Españoles: Grupo de Didáctica de la Geografía (AGE), con una frecuencia anual desde 2002, y consta de dos números; revista
Encontramos un total de 388 registros en esta primera exploración, de los cuales descartamos las revistas que no tienen formato físico y que no se encuentran en las bibliotecas de Bogotá, los trabajos de grado -sin publicación-, los documentos políticos y legislativos y los documentos electrónicos. Una vez realizado este ejercicio quedamos con 108 documentos que constituyen una base de datos de 262 registros, en los cuales se incluyen los artículos de revistas y los capítulos de libro. De tal manera que el trabajo que presentamos, es una muestra significativa -no representativa desde enfoques estadísticosde la producción en el campo. Teniendo en cuenta las especificidades de esta documentación se construyó una base de datos a partir de tres criterios generales de diseño, ${ }^{9}$ siete módulos de información ${ }^{10}$ y treinta $\mathrm{y}$ siete campos de almacenamiento de datos ${ }^{11}$.

Iber: didáctica de las ciencias sociales, geografía e historia, de editorial Graó, con una frecuencia trimestral desde 1994, consta de 46 números y del resumen de algunos de sus artículos; Revista de Teoría y Didáctica de las Ciencias Sociales, de la Facultad de Humanidades y Educación de la Universidad de los Andes, con una frecuencia anual desde 1996, y consta de once números. Esta última revista tiene versión digital en: http://www.saber.ula.ve/gitdcs/. El total de revistas editadas en el período es de 58 , de las cuales solamente se tiene acceso en medio físico a ocho en los centros de documentación explorados.

9 Los criterios de diseño tienen que ver con a los usos que se pueden derivar del diseño de la base de datos; para este caso se definieron tres tipos de criterios: registro de la información -se refiere a los distintos módulos y campos requeridos para almacenar los datos correspondientes al volumen documental del estado del arte-; administración de la base de datos -corresponde a las posibilidades de ingresar, editar o ampliar la información de los registros-; y tipos de consulta -modalidades de búsqueda que pueden realizarse en el conjunto de registros ingresados a la base de datos-.

10 Los módulos de información se componen de los datos básicos del registro ingresado. El primer módulo clasifica el documento; el segundo, detalla la información básica de su edición; el tercero, identifica y clasifica los autores del documento; el cuarto, describe sintéticamente los contenidos correspondientes al registro; el quinto, cataloga el documento de acuerdo con los descriptores que se definieron en el proceso de sistematización de la información y presenta los conceptos centrales de los documentos reseñados; el sexto, contiene las fuentes sobre enseñanza y aprendizaje de las ciencias sociales referenciadas en los registros, y el séptimo, ubica al usuario de la base de datos en relación con el centro de documentación en el cual puede tener acceso al documento registrado.

11 En cuanto a los campos, éstos son espacios para diligenciar los datos requeridos, los cuales describen la información o la clasifican en una lista desplegable o en una ventana Popus. 
Para el desarrollo de esta base de datos se diseñó una aplicación bajo un ambiente web con las siguientes herramientas: a). el motor de base de datos MySQL, distribuido bajo la licencia GPL, caracterizado por tener un código abierto y una capacidad de funcionamiento en distintas plataformas como Windows o Linux con idénticos resultados. b). Lenguaje de programación para páginas dinámicas PHP -Hyper Text Pre-processor-, distribuido bajo licencia libre, funciona en múltiples plataformas o sistemas operativos y múltiples servidores web y está desarrollada con las mismas herramientas del portal de la Universidad Pedagógica Nacional. Estas especificidades técnicas permiten que la base de datos sirva como mecanismo de consulta sobre la información acopiada hasta el momento, para la indagación de fuentes especializadas en la enseñanza y aprendizaje de las ciencias sociales, la formulación de proyectos de investigación y la consulta que puedan hacer estudiantes y docentes en el portal de la Universidad Pedagógica Nacional (http://pedagog. webcindario.com/index.php).

\section{Las ciencias sociales en contextos escolares: su campo discursivo}

Una caracterización general de la documentación registrada mostró las tendencias en cuanto a tipo de material12, sitios de publicación ${ }^{13}$, editoriales e instituciones que financian proyectos de investigación e innovación ${ }^{14} \mathrm{y}$ autores o grupos de investigación

12 La descripción general de la documentación corresponde a los documentos ajustados a los criterios desde los cuales se delimitó la información. En este proceso se concretó el análisis sobre un total de 262 registros, compuestos por 75 libros, 79 capítulos de libros, 24 memorias de congresos y eventos académicos y 29 documentos inéditos, producto de la financiación en convocatorias diseñadas por organismos estatales. Estos documentos se clasificaron en dos tipos de materiales: las investigaciones o innovaciones, que corresponden al $56 \%$, y los ensayos, que corresponden al $44 \%$. La difusión de las investigaciones se hace preferiblemente por medio de la publicación de libros $(30 \%)$ y la de los ensayos ocurre fundamentalmente mediante revistas especializadas (32\%).

13 Los sitios de publicación de esta documentación son en su mayor parte ciudades españolas, las cuales constituyen el $44,27 \%$ de los registros; luego siguen Colombia, con un $29 \%$ y Argentina, con el $22 \%$, de la producción; los demás registros se concentran en México y Venezuela.

14 Las editoriales se agruparon en seis grupos: editoriales comerciales, $65 \%$; editoriales universitarias y de centros de investigación, 30\%; representativos en el campo ${ }^{15}$. En cuanto a las temáticas desarrolladas en los documentos registrados, diseñamos 22 descriptores, delimitados a partir de la información acopiada y formalizados para ubicar los conceptos claves o centrales, a partir de la estructura jerárquica propuesta por el Tesaurus de la Unesco (2002). El conjunto de aspectos de esta caracterización permite identificar las condiciones de producción académica del campo de la enseñanza y el aprendizaje de las ciencias sociales, pero consideramos que es importante enfatizar en los campos temáticos ubicados en los 22 descriptores, pues allí se hacen visibles los enunciados que configuran este campo.

Siguiendo la estructura propuesta por el Tesaurus de la Unesco, formulamos siete descriptores para la primera relación jerárquica de la documentación, referida al tema o problema central abordado en el documento:

- Disciplinas sociales. Discusiones actuales en el ámbito epistemológico de las ciencias sociales que formulan hipótesis, tesis, debates, análisis o reflexiones sobre las posibles relaciones que se pueden establecer entre estos campos disciplinares y su enseñanza escolar.

- Disciplinas escolares. Saberes escolares que han configurado el área de ciencias sociales (historia, geografía, economía, ciencias humanas, ciencias políticas).

- Didácticas específicas. Dinámicas de configuración del campo de la didáctica de las ciencias sociales a partir de sus discusiones epistemológicas, conceptuales y metodológicas.

- Innovaciones. Propuestas de enseñanza que buscan apartarse de formas de trabajo convencionales.

organismos de cooperación internacional, $6 \%$; documentos publicados por distintas organizaciones, asociaciones o instituciones asociadas con fines de divulgación, $5 \%$; instituciones y organismos estatales, $5 \%$, y por último, materiales inéditos, $4 \%$.

15 En la información acopiada están registrados 283 autores, clasificados de acuerdo con el país de procedencia y la frecuencia de su publicación. En relación con el país de procedencia, se encontró que el $43 \%$ de los autores del campo son españoles, el $30 \%$ colombianos y el $20 \%$ argentinos. Dentro de este conjunto de autores se ubicaron los más representativos del volumen documental y los grupos de investigación. 
- Aprendizaje de las ciencias sociales. Distintos tipos de procesos cognitivos que se emplean o desarrollan, cuando los estudiantes escolares abordan las ciencias sociales.

- Enseñanza de las ciencias sociales: Tipos de prácticas escolares que se realizan con los estudiantes al abordar las ciencias sociales.

- Tendencias pedagógicas. Corrientes de pensamiento pedagógico abordadas para desarrollar una temática o problemática sobre la enseñanza y el aprendizaje de las ciencias sociales.

Después de clasificar los registros en estas siete categorías, se ubicaron los documentos en uno o varios de los descriptores de la Segunda relación jerárquica, caracterizada por los aspectos desde los cuales se desarrolla el tema o problema central que se aborda en el documento registrado:

- Currículo. Criterios de legitimación de la selección, clasificación y seriación de contenidos y prácticas escolares en ciencias sociales.

- Formación de maestros. Niveles y prácticas de formación de los profesionales en educación en ciencias sociales.

- Textos escolares. Materiales impresos por casas editoriales comerciales de reconocido prestigio o por imprentas estatales, a partir de los cuales se desarrolla el currículo hegemónico en ciencias sociales.

- Saberes y prácticas de enseñanza. Experiencia del maestro de ciencias sociales proveniente de su ejercicio docente.

- Problemáticas escolares. Distintas tensiones que ocurren en el escenario escolar, de carácter psicológico, pedagógico, didáctico, curricular o regulativo, las cuales se identifican y conceptualizan a partir de su problematización, reflexión o sistematización.

- Reformas educativas. Mecanismos y políticas de administración y gobierno del sector educativo, que se focalizan en las ciencias sociales escolares.

- Diseño de unidades y actividades. Contenidos derivados de las ciencias sociales y propuestas metodológicas que resultan de un ejercicio de investigación, innovación o sistematización de experiencias, que se organizan y clasifican en módulos temáticos.

Después de catalogar los registros en los descriptores de segunda jerarquía, se clasificaron en uno o varios de los descriptores denominados Términos asociados, referidos al tipo de campo temático con el cual se puede relacionar el documento registrado:

- Problemas sociales del contexto. Situaciones de carácter local o nacional que afectan la enseñanza y el aprendizaje de las ciencias sociales escolares o que se incorporan al currículo de ciencias sociales en el ámbito escolar.

- Problemas sociales contemporáneos. Situaciones de carácter global que afectan la enseñanza y el aprendizaje de las ciencias sociales escolares o que se incorporan al currículo de ciencias sociales en el ámbito escolar.

- Sistema educativo nacional. Organización y administración del sector educativo en todos sus niveles y modalidades.

- Profesión docente. Formación y ejercicio calificado de la enseñanza en distintos niveles y modalidades educativas.

- Producción de materiales. Textos, cartillas, módulos, multimedia, producto en línea, base de datos y software, diseñados, desarrollados y empleados para abordar problemáticas educativas o llevar a cabo procesos de enseñanza.

- Contexto educativo. Caracterización del ámbito educativo a escala local, nacional o global, análisis de sus dinámicas discursivas e incidencia en las prácticas educativas y escolares cotidianas.

- Contexto político. Caracterización del ámbito político a escala local, nacional o global, análisis de sus dinámicas discursivas e incidencia en las prácticas educativas y escolares cotidianas.

- Contexto cultural. Caracterización del ámbito cultural a escala local, nacional o global, análisis de sus dinámicas discursivas e incidencia en las prácticas educativas y escolares cotidianas.

El primer nivel de análisis consistió en establecer las tendencias temáticas en la primera relación jerárquica de categorías. Encontramos que prevalecen los trabajos que enfatizan en los campos disciplinares y sus procesos de adecuación en la enseñanza escolar 
(disciplinas sociales: $31 \%$ ) y en la investigación sobre la enseñanza desde experiencias específicas (enseñanza de las ciencias sociales: 24\%). En un menor número de documentos abordan las especificidades de la didáctica de las ciencias sociales (didácticas específicas: 13\%) o que formulan propuestas de trabajo pedagógico alternativas en la enseñanza o el aprendizaje de las ciencias sociales (innovaciones: $15 \%)$. El grupo más reducido de trabajos analiza las ciencias sociales y su relación con el campo pedagógico (tendencias pedagógicas: 7\%), con el saber escolar (disciplinas escolares: 5\%) o con el aprendizaje (aprendizaje de las ciencias sociales: $4 \%$ ).

Las problemáticas centrales de los documentos trabajados muestran que la enseñanza de las ciencias sociales está fuertemente influenciada por las disciplinas sociales desde una perspectiva regulativa y normativa, pues se indican los conceptos, nociones, estructuras o temáticas que se deben enseñar, sin reflexionar sobre las especificidades del saber escolar o de las condiciones institucionales en las cuales ocurren las prácticas de enseñanza y se constituye el código disciplinar (R. Cuesta, 1997).

Así mismo, existe un alto número de trabajos que basan sus conclusiones en investigaciones realizadas con grupos de estudiantes escolares, a partir de diseños metodológicos que buscan comprobar hipótesis, producir experiencias exitosas o proponer modelos de enseñanza para transferir experiencias a contextos diferentes sobre el supuesto de la duplicación de resultados satisfactorios. La dispersión de los otros trabajos muestra una constitución débil en asuntos estratégicos del campo (A. Camilloni, 1996), como la configuración de las ciencias sociales escolares (G. Travé, 1998), la reducción de la enseñanza a la sistematización aislada de experiencias inscritas en el campo hegemónico de la educación (competencias, indicadores de logro, integración de las áreas) y la subordinación del conocimiento sobre los sujetos escolares, al aprendizaje como categoría psicológica (J. Larrosa, 1995).

El segundo nivel de análisis consistió en identificar la ocurrencia -número de veces que aparece un descriptor en los documentos- y coocurrencia -número de veces que cada descriptor está asociado con otro descriptor- temática de la documentación acopiada y clasificada. Para realizar este análisis utilizamos la teoría actor-red, que articula un conjunto de conceptos que le otorgan un sentido estratégico, en el marco de las actuales condiciones de producción de información, ya que mediante sofisticados esquemas de graficación de los enunciados que constituyen un campo de discurso, visibilizan la metáfora espacial que desarrollaron M. Foucault (1966) y P. Bourdieu (1997) y que aplicaron B. Berstein (1993) y M. Díaz (1993) en el ámbito pedagógico, para mostrar las relaciones de poder que se establecen entre los agentes y agencias en un campo discursivo. En la perspectiva de la teoría actor-red, el actor (agente o agencia), tiene múltiples formas de aparición (autor, libro, revista, enunciado) y lo que interesa es mirar las relaciones que establece y el lugar estratégico en el que se posiciona en el campo discursivo del cual hace parte (M. Callon, J. P. Courtial y H. Penan, 1995). Para graficar las relaciones entre los enunciados utilizamos el software Leximappe, que se constituye en uno de los desarrollos tecnológicos de la teoría actor-red16.

$\mathrm{Al}$ establecer todas las relaciones posibles, se puede mostrar cuántas veces aparece clasificado un documento bajo un descriptor, y a su vez, cuántas veces dicho descriptor está asociado con los demás descriptores en todos los otros documentos que forman parte de la base documental del estado del arte. La frecuencia con la cual estos descriptores

16 En este software se emplea una técnica híbrida que combina umbrales con algoritmos iterativos. Puede imponerse un umbral mínimo de ocurrencia y de coocurrencia de las palabras, así como un valor mínimo y otro máximo del tamaño de las agrupaciones o temas obtenidos, que nos permite generar agrupaciones de estructura libre 0 en forma de estrella. Leximappe combina simultáneamente las técnicas con umbral

-máximos y mínimos de ocurrencia y coocurrencia de los descriptores-y las de enlace -los vínculos entre descriptores-, aportando la posibilidad de utilizar índices como el de cohesión interna o densidad y el de cohesión externa o centralidad. Otro importante desarrollo tecnológico lo constituye el programa Redes versión 2005, que permite graficar interacciones a partir del contenido de documentos textuales de cualquier tipo: artículos, tesis, informes, libros, entre otros. Este programa se basa también en la ciencimetría de redes según la teoría actor - red y emplea como instrumento cuantitativo el análisis de palabras asociadas. 
están asociados configura nodos centrales en el campo discursivo trabajado. Aplicando este desarrollo tecnológico, los nodos centrales en el campo de la enseñanza y el aprendizaje de las ciencias sociales son: sistema educativo nacional, diseño de unidades $y$ actividades y contexto educativo. Estos nodos se pueden graficar en un diagrama compuesto por dos ejes: el eje X representa la intensidad de las relaciones entre los nodos -centralidad-y caracteriza el campo de la enseñanza y el aprendizaje de las ciencias sociales en el período estudiado. El eje Y representa la intensidad de las relaciones en el interior de cada nodo - densidad-y caracteriza el nivel de consolidación de las discusiones básicas del campo. Este gráfico se denomina diagrama estratégico y permite ubicar las posiciones relativas dentro de la red de los descriptores y sus transformaciones en un lapso específico (figura 1 ).

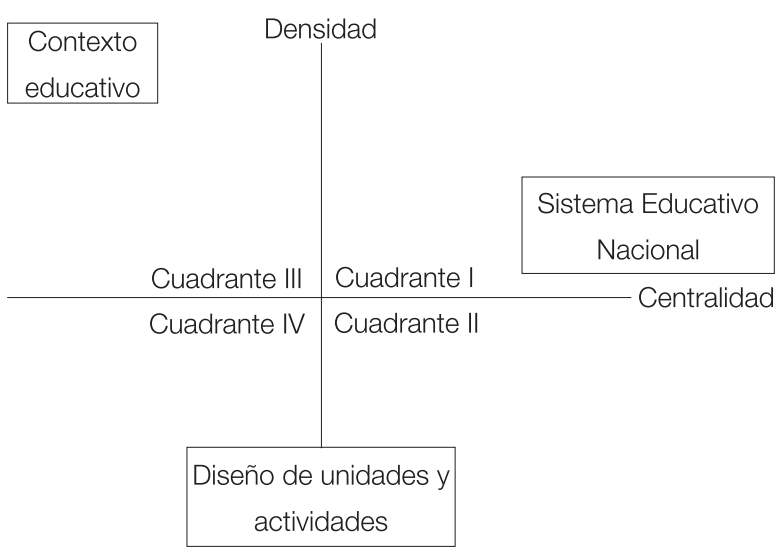

Figura 1. Diagrama estratégico del campo discursivo de la enseñanza y aprendizaje de las ciencias sociales.

El nodo sistema educativo nacional se localiza en el primer cuadrante y se caracteriza por su elevada centralidad y su baja densidad, lo cual indica que tiene relaciones fuertes con los nodos y relaciones débiles con los descriptores internos que lo constituyen en nodo, mostrándonos que es una temática que genera vínculos y relaciones en el campo, pero que aún está por desarrollarse. El nodo diseño de unidades y actividades, que se encuentra entre el segundo y el cuarto cuadrantes se caracteriza por su mínima densidad y baja centralidad, lo cual indica que tiene relaciones débiles con los nodos del diagrama y escaso nivel de asociación con los descriptores internos; esto evidencia que es una temática sin desarrollo interno, que presenta algunas relaciones con las temáticas centrales del campo. Por último, el nodo contexto educativo se encuentra en el tercer cuadrante y se caracteriza por su alta densidad y baja centralidad, lo cual indica que cuenta con relaciones fuertes de los descriptores internos que lo componen y relaciones débiles con los otros nodos; esto señalaría el desarrollo de una temática especializada y sin relación con los demás temas del campo. La descripción interna de cada uno de los nodos centrales nos permite identificar las tendencias temáticas y los énfasis que se han ido configurando en el campo.

\section{Contexto educativo}

El descriptor contexto educativo incluye documentos en los que abordan aspectos relacionados con el ámbito educativo a nivel local, nacional o global tanto en sus dinámicas discursivas como en su incidencia en las prácticas escolares. Este descriptor conforma una red de relaciones con otros seis descriptores (figura 2), donde se presentan las asociaciones más fuertes.

El nodo contexto educativo corresponde a la última clasificación jerárquica (términos asociados) y se convierte en la categoría externa más importante por ser el campo discursivo de inscripción del discurso pedagógico y didáctico. Su presencia en el diagrama estratégico muestra que los trabajos en disciplinas sociales, disciplinas escolares, didácticas específicas, innovaciones, aprendizaje de las ciencias sociales, enseñanza de las ciencias sociales y tendencias pedagógicas (descriptores de la primera relación jerárquica) como áreas de investigación, no se relacionan con el campo conceptual de su procedencia. Esto hace que el contexto educativo sea altamente autorreferenciado, a la vez que explica la poca incidencia que tiene en las prácticas educativas y escolares cotidianas.

De acuerdo con el número de asociaciones entre los descriptores correspondientes al nodo contexto 


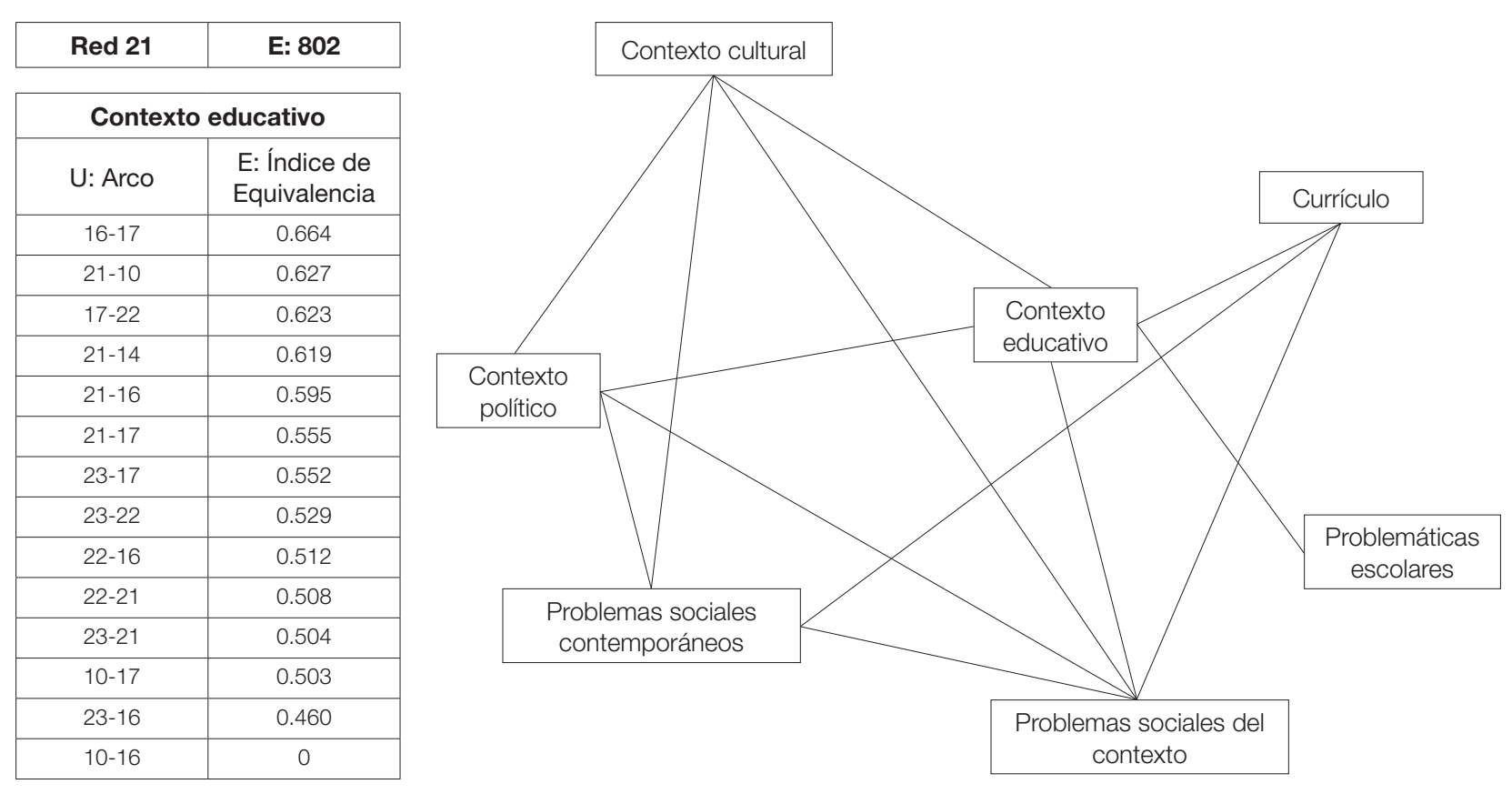

Figura 2. Redes internas del nodo contexto educativo.

educativo (21)17, se pueden establecer las temáticas que constituyen vínculos centrales de la red interna. El primer grupo lo conforman los descriptores que se asocian de cuatro a cinco veces: problemas sociales contemporáneos (17), problemas sociales del contexto (16), contexto cultural (23) y contexto político (22). Las temáticas generales a las cuales se refieren los trabajos inscritos en esta asociación de enunciados corresponden a las relaciones de género y la articulación de las TIC con la enseñanza y el aprendizaje de las ciencias sociales; a los procesos de enseñanza en la perspectiva de la formación ciudadana y de la enseñanza de los derechos humanos; a la educación bilingüe o multilingüe en ámbitos culturales diversos o en los que se incluyen cátedras transversales, como la de afrocolombianidad, y por último a los estudios sobre la ejecución de reformas educativas enfocadas al ámbito de la evaluación o las competencias.

17 Este número aparece frente a los 22 descriptores y corresponde al código asignado a cada enunciado, para señalar la intensidad de las asociaciones en la tabla adjunta a cada uno de los gráficos.
El segundo grupo lo conforman los descriptores que se asocian de dos a tres veces: problemas sociales contemporáneos (17), problemas sociales del contexto (16) y currículo (10). En este grupo se ubican los trabajos que enfatizan en la adecuación de temáticas relacionadas con el género, las TIC, la familia, los valores, la convivencia o la ciudad a los contenidos escolares. Finalmente, en el tercer grupo se encuentra el descriptor problemáticas escolares (14), asociado sólo con el descriptor del nodo contexto educativo (21), en el cual se encuentran trabajos en los que se diagnostican situaciones escolares precisas para el desarrollo de propuestas para la enseñanza. La dinámica general de este nodo muestra que a pesar de la creciente preocupación por problemáticas que se consideran de vanguardia, estos estudios no están vinculados a las prácticas de enseñanza, puesto que esta relación ocurre únicamente en las propuestas que tratan de adecuar contenidos derivados de esas problemáticas al diseño curricular. 


\section{Diseño de unidades y actividades}

La segunda red interna que se forma en el diagrama estratégico es la del nodo diseño de unidades y actividades (24), el cual agrupa documentos en los cuales se divulgan propuestas para la enseñanza, organizadas en módulos temáticos compuestos por los siguientes aspectos: contenidos, metodologías, objetivos de enseñanza y aprendizaje, materiales didácticos e indicaciones precisas para la evaluación (figura 3).

\begin{tabular}{|c|c|}
\hline Red 24 & E: 456 \\
\begin{tabular}{|c|c|}
\hline \multicolumn{2}{|c|}{$\begin{array}{c}\text { Diseño de unidades } \mathbf{y} \\
\text { actividades }\end{array}$} \\
\hline U: Arco & $\begin{array}{c}\text { E: Índice de } \\
\text { Equivalencia }\end{array}$ \\
\hline $13-4$ & 0.350 \\
\hline $13-19$ & 0.288 \\
\hline $4-19$ & 0.238 \\
\hline $13-24$ & 0.149 \\
\hline $24-4$ & 0.142 \\
\hline $24-19$ & 0.93 \\
\hline $24-3$ & 0.31 \\
\hline $3-19$ & 0.12 \\
\hline $7-13$ & 0.11 \\
\hline $9-24$ & 0.10 \\
\hline $7-24$ & 0 \\
\hline $13-3$ & 0 \\
\hline
\end{tabular}
\end{tabular}

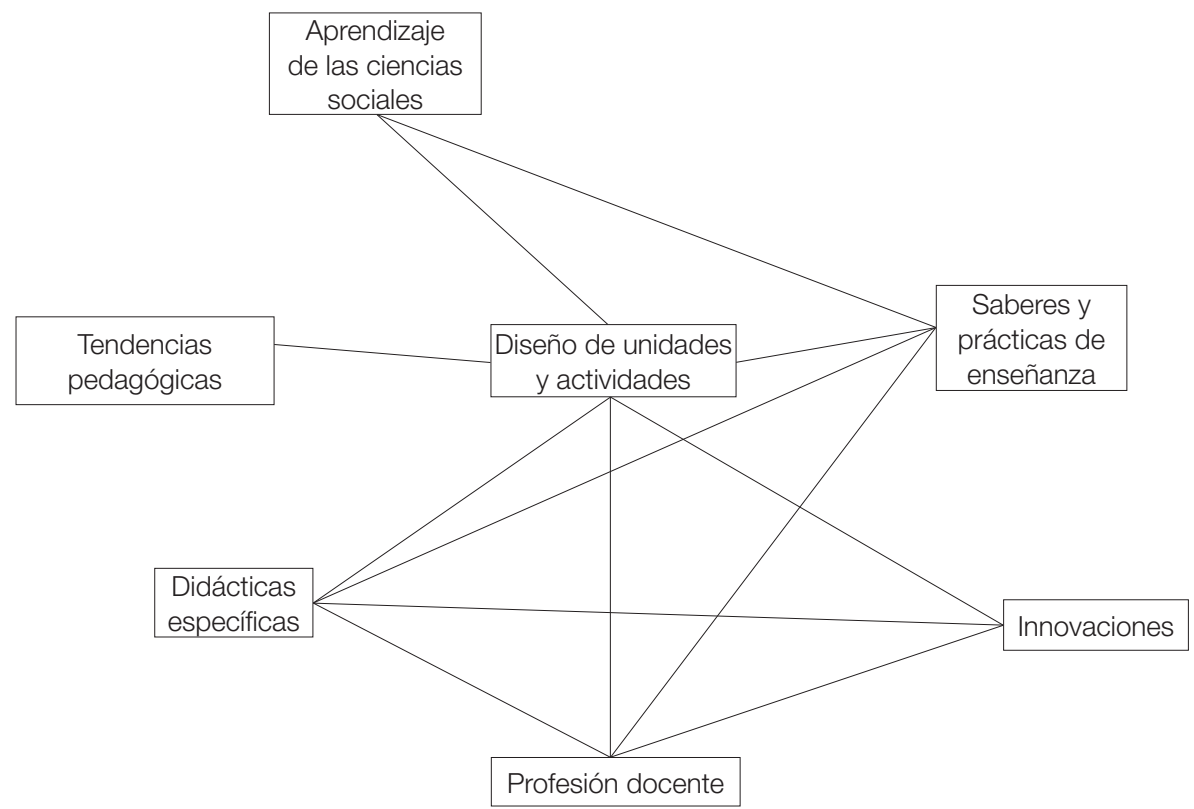

Figura 3. Redes internas del nodo diseño de unidades y actividades.

Este descriptor corresponde a la segunda relación jerárquica, en la cual se encuentran los temas que caracterizan el campo discursivo de la enseñanza y el aprendizaje de las ciencias sociales. El nodo que conforma el descriptor diseño de unidades y actividades presenta una mínima asociación con los descriptores de la primera relación jerárquica, relacionada con la investigación y los aportes conceptuales sobre didáctica de las ciencias sociales. Según el número de asociaciones las agrupaciones temáticas de este nodo son las siguientes: el primer grupo de enunciados se asocia de cuatro a cinco veces: saberes $y$ prácticas de enseñanza (13), profesión docente (19) y didácticas específicas (3). Las temáticas generales a las que se refieren los trabajos inscritos en este grupo sistematizan experiencias de enseñanza, en el marco de programas de formación docente o de proyectos que buscan recuperar el saber pedagógico de los docentes y estudios que buscan precisar el desarrollo conceptual de la didáctica específica de las ciencias sociales.

El segundo grupo de enunciados se asocia de dos a tres veces: innovaciones (4) y aprendizaje de las ciencias sociales (7). En este grupo se encuentran trabajos que buscan generar alternativas de enseñanza en el marco de proyectos de investigación e innovación, en la perspectiva de optimizar de los procesos de aprendizaje. Por último, se encuentra el enunciado tendencias pedagógicas (9), asociado solamente con el descriptor del nodo diseño de unidades y actividades (21), en el cual se ubican trabajos que diseñan materiales en función de un modelo o tendencia pedagógica, más que en relación con indicadores de aprendizaje o de evaluación. Los grupos que se conforman en este nodo muestran que la producción de material educativo es una preocupación 
central en el campo discursivo estudiado, pero dicha producción no se relaciona con la pedagogía, que es el saber de referencia, y se relaciona muy poco con la didáctica y sus conceptos básicos.

\section{Sistema educativo nacional}

La tercera red que se forma en el diagrama estratégico es la del descriptor sistema educativo nacional
(18), en el cual se ubican los documentos que hacen referencia a la organización, gestión y administración del sector educativo en sus diferentes modalidades. Este descriptor corresponde a los términos asociados, que se encuentran en una tercera relación jerárquica, que indica la relación externa del campo (figura 4).

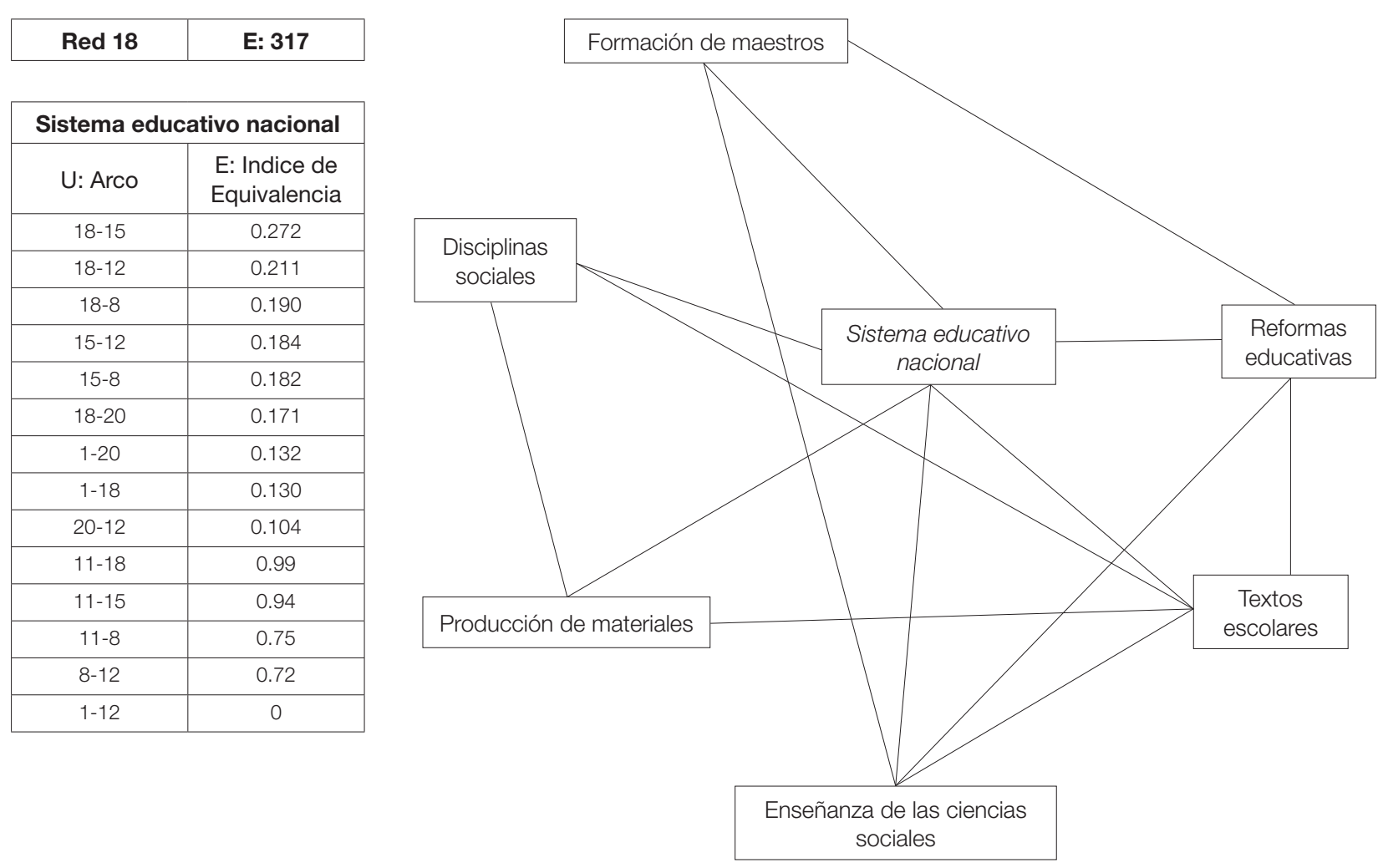

Figura 4. Redes internas del nodo sistema educativo nacional.

Esto nos muestra que el desarrollo alcanzado por temáticas como reforma educativa, análisis de textos escolares y formación de maestros es aún incipiente e incide en la poca relación que se establece entre la formalización de los procesos analíticos y reflexivos, a los que podrían conducir las temáticas. Según el número de asociaciones, las agrupaciones temáticas de este nodo son las siguientes: el primer grupo lo integran los descriptores que se asocian de cuatro a cinco veces: textos escolares (12), reformas educativas (15) y enseñanza de las ciencias sociales (8). Los estudios ubicados en esta agrupación ana- lizan los textos escolares y las reformas educativas nacionales, con el propósito de generar propuestas de enseñanza para la integración de los países del Convenio Andrés Bello, de la Organización de Estados Iberoamericanos o de la Comunidad Andina de Naciones. El segundo grupo está conformado por los descriptores que se asocian de dos a tres veces: producción de materiales (20), disciplinas sociales (1) y formación de maestros (11).

Este grupo consta de trabajos en los cuales se analiza la producción de textos escolares y materiales de divulgación educativa, los programas de 
formación profesional de docentes o que buscan adecuar contenidos disciplinares a materiales educativos, en la perspectiva de producir materiales o generar propuestas de formación acordes con los programas nacionales propuestos por las entidades gubernamentales o programas de integración binacional, regional o iberoamericana. Las agrupaciones muestran de este modo que la formación y la producción de materiales de amplia divulgación las promueven y financian organismos nacionales e internacionales que buscan ejecutar sus propuestas políticas de integración, sin que existan relaciones de estos proyectos con la producción académica en los campos de la pedagogía y la didáctica.

\section{La emergencia de la didáctica de las ciencias sociales: ¿campo en consolidación o en disgregación?}

La localización del campo discursivo ${ }^{18}$ de la enseñanza, y el aprendizaje de las ciencias sociales en los enunciados, nodos y relaciones que acabamos de exponer, especifica las particularidades de dicho campo, con respecto a los procedimientos externos que lo controlan y los procedimientos internos que lo clasifican y ordenan. Los procedimientos externos de control del discurso ${ }^{19}$ buscan someter este campo de saber eludiendo las particularidades en las que ocurren los acontecimientos escolares: contexto educativo y sistema educativo nacional, y los procedimientos internos 20 ordenan los enunciados que particularizan este campo de saber en relación con otros campos, en este caso conformados para la enseñanza de las disciplinas escolares.

18 Hacer visible el campo discursivo implica "describir unos enunciados, describir la función enunciativa de que son portadores, analizar las condiciones en que se ejerce esta función, recorrer los diferentes dominios que supone la manera en que se articulan", es "sacar a la luz lo que podría individualizarse como formación discursiva" (Foucault, 1966, 196).

19 Según Foucault (1971), estos procedimientos externos son lo prohibido, la separación y rechazo, y la oposición entre lo verdadero y lo falso, como regímenes de producción de verdad.

20 Los procedimientos internos, según Foucault (1971), son comentario, autor y disciplina como principios de ordenación, clasificación y actualización permanente.

\section{El nodo contexto educativo como procedimiento externo de control discursivo}

El contexto educativo se asemeja a lo que M. Díaz (1993) denomina campo intelectual de la educación, constituido por las relaciones, posiciones y prácticas, que expresan las dinámicas y tensiones de los intelectuales en la producción discursiva, y caracterizado por un cierto nivel de autonomía del campo del Estado y del campo pedagógico. Allí se producen enunciados determinantes - de alta densidad en el diagrama estratégico- que contribuyen a la naturalización de determinados enunciados como si fueran necesarios y no requirieran discusión. Entre las categorías y líneas de trabajo que hoy son estructurantes de este campo se encuentran la incorporación de las TIC en las prácticas educativas y en los procesos de formación de docentes, la interculturalidad y las prácticas de educación multilingüe en contextos escolares; la ciudadanía, la ética y los valores, como formulaciones estratégicas orientadas a educar para el trabajo mediante el desarrollo de competencias. Estos son los imperativos del campo educativo y es lo que define el interés de los organismos multilaterales por promover la reflexión en estos campos y la viabilidad de los proyectos de edición que se presentan en las editoriales comerciales. En este caso la hegemonía de ciertas temáticas está ligada con las dinámicas de una cultura global que promueve cada vez con mayor eficacia un interés consensuado por ciertos temas, entre los que se encuentran los ya anotados.

Las débiles relaciones de contexto educativo con los otros dos nodos del esquema estratégico indican que los descriptores de la primera relación jerárquica, como áreas de investigación, no se relacionan con el campo conceptual de su procedencia. Esto hace que el contexto educativo sea altamente autorreferenciado, lo cual podría poner en debate la hipótesis de los campos de recontextualización de los enunciados provenientes del campo intelectual de la educación -oficial y pedagógico- propuestos por M. Díaz (1993) y se aproxima más a la idea de órdenes discursivos independientes que de acuerdo con sus dinámicas internas son discontinuos y, en ocasiones, yuxtapuestos (S. Gvirtz, S. Larripa y Á. Oría, 2004). 


\section{El nodo sistema educativo nacional como procedimiento externo de control discursivo}

La ubicación del sistema educativo nacional como enunciado de baja densidad, es decir, de poca fuerza en las relaciones que lo constituyen como nodo, muestra que los intereses de los entes financiadores, de los programas de formación y de la institución escolar, se conjugan en la necesidad de hacer coincidir en una sola formulación el cumplimiento administrativo de las reformas educativas, sin que estas últimas en sí mismas sean objeto de análisis y reflexión. Así como con la aparición de los procesos de escolarización a principios del siglo XX se buscaba generar un sistema para poner en funcionamiento la simultaneidad sistémica de Comenio ( $M$. Narodowski, 1999), así se busca desde los sistemas educativos nacionales legitimar enunciados que pongan en funcionamiento estrategias, ahora globales, de formación. Los temas nodales del contexto educativo se intentan adecuar a las condiciones de un sistema que cada vez es menos nacional y más regional, anclado a los proyectos culturales hegemónicos de los organismos multilaterales o a los requerimientos del mercado educativo (A. Pérez, 2000).

\section{El nodo diseño de unidades y actividades como procedimiento interno de control discursivo}

De los descriptores internos del volumen documental, el que se configuró como un nodo en la red del diagrama estratégico fue el correspondiente al diseño de unidades y actividades, lo cual evidencia que los procesos de reflexión y de investigación e innovación están sujetos a un principio de aplicabilidad en la enseñanza. Esta concepción proviene, a nuestro juicio, de dos posiciones muy difundidas en el ámbito de la producción académica referida a contextos escolares: la primera tiene que ver con una concepción sobre teoría y práctica, desde la cual el supuesto que se agencia es que la elaboración teórica tiene unos niveles de aplicación en la práctica y que la evaluación de la práctica debe estar sujeta a los supuestos de dicha teoría. Entre estas dos dimensiones del conocimiento no se establece una relación reflexiva sino una relación causal de aplicabilidad.
Por tal razón, aunque predominen las investigaciones de corte cualitativo, particularmente derivadas de esquemas de investigación acción participación, la concepción epistemológica que soporta estas investigaciones proviene de esquemas experimentales, desde los cuales lo fundamental es comprobar la teoría y mostrar que la experiencia particular y personal es la mejor frente a otras propuestas, es legitimado todo esto por los programas de formación profesional y continuada, o las que son premiadas o galardonadas por entidades estatales.

La otra razón es el peso específico que tienen disciplinas como la psicología, frente a las disciplinas escolares o las disciplinas sociales, esto es, el supuesto que considera que lo cognitivo, el aprendizaje y los procesos de pensamiento son un territorio exclusivo de la psicología ${ }^{21}$ y que frente a éstos no existen contribuciones desde la pedagogía, la sociología, la antropología, la lingüística y la filosofía que puedan aportar conclusiones complementarias o incluso divergentes. Este peso de la psicología ha hecho que los resultados de las investigaciones estén sujetos a la composición de esquemas de trabajo que subordinan la enseñanza como actividad intencionada de un sujeto específico, el maestro, a los presupuestos del aprendizaje derivado de la psicología. Los materiales educativos que provienen de concepciones de esta naturaleza se formulan sin reflexiones sobre el currículo, las disciplinas escolares, las dinámicas de la escuela contemporánea y los contextos locales no estereotipados.

Esto explica que las propuestas didácticas más difundidas y demandadas desde mediados de la década de los noventa busquen optimizar los procesos de aprendizaje mediante la adopción o incorporación de códigos que garanticen enseñanza efectiva, sin que medien ejercicios de análisis sistemático, sobre el contexto educativo, político

21 En la investigación de la cual procede este artículo el análisis sobre autores muestra que prevalecen en importancia y número los autores que provienen de campos relacionados con la psicología. Ver Informe de investigación. Estado del arte en la enseñanza y el aprendizaje de las ciencias sociales: producción académica en los países de habla hispana. 1990-2000. Avalado y financiado por el Ciup-UPN entre 2004 y 2006. 
y cultural, sobre el saber que se enseña en relación con el campo disciplinar o temático de referencia, o sobre la experiencia de los profesores en el área de su especialidad22. En busca de la eficiencia en el resultado educativo, la enseñanza ha transitado de lo que denomina M. Narodowski (1999) el ideal pansófico de enseñar todo a todos y de la "utopía sociopolítica" de la formación de un cierto tipo de hombre, en una perspectiva moderna, universalista, hacia las "nuevas utopías hiperadaptativas", caracterizadas ya no por el para qué de la enseñanza, sino por el cómo, pregunta propia de la instrumentalización del saber. Dicha transformación en las prácticas de enseñanza hace que los maestros vivan “... en medio de una proliferación de modas, polución de posiciones, vertientes, soluciones y posturas pedagógicas..." que imponen una elección a la carta de “... los métodos didácticos que más convienen a nuestra actividad educadora: la coherencia ideológica y el encadenamiento totalizador con finalidades políticas naufraga frente a la búsqueda desesperada de efectividad" (M. Narodowski, 1999, 33).

En este sentido, la reflexión sobre los efectos políticos de un cierto tipo de enseñanza, sobre la selección de los contenidos que se llevan a la clase y sobre la didáctica en su campo de referencia más amplio -la pedagogía-, no se realiza sino que se deja en manos de la administración pública, conozca o no las circunstancias del contexto educativo o las discusiones del campo pedagógico. Esta situación se produce en medio de un amplio debate sobre el carácter de didáctica específica de las ciencias sociales en el marco de la didáctica general, en el ámbito discursivo de la pedagogía y en las dinámicas del contexto educativo.

Un tercer grupo de procedimientos de control discursivo, denominado condiciones de utilización

22 Es importante señalar que la convocatoria del Idep en Bogotá, durante el 2002, permitió sistematizar experiencias de innovacióninvestigación en el aula, realizadas por los docentes. Sin embargo, estas iniciativas de financiación siguen siendo parciales y localizadas. del discurso ${ }^{23}$, se refiere al acceso o exclusión de los sujetos a ciertos discursos, que se caracterizan por códigos de clausura, frente a otros discursos y otros sujetos. En este conjunto de procedimientos Foucault ubica la educación como procedimiento de adecuación social del discurso, mediante el cual "todo individuo en una sociedad como la nuestra puede acceder a no importa qué tipo de discurso" (Foucault, 1971,36), con tal que se constituya en "la forma política de mantener o de modificar la adecuación de los discursos con los saberes y los poderes que implican (Ibid., 37)". Este tercer procedimiento de control discursivo permite aproximarse a la tensión entre la consolidación y la disgregación de la didáctica de las ciencias sociales, en tanto que los enunciados que conforman los procedimientos externos -contexto educativo y sistema educativo nacional- buscan la institucionalización de un saber (J. Sauquillo, 1989), que prefieren mantener sometido y circunscrito mediante procedimientos de adecuación discursiva. - Diseño de unidades y actividades-.

El lugar que ocupan los enunciados centrales del campo de la didáctica de las ciencias sociales está subordinado a una jerarquía mayor, impuesta por los enunciados de primer orden, que en nuestro análisis constituyen los procedimientos externos de control discursivo. Dichos procedimientos validan y "dan prioridad a los discursos universales y globales sobre ciertos saberes sometidos o relegados al olvido, mediante un dispositivo de jerarquización del saber" (J. Sauquillo, 1989, 276), en el cual, para el caso que nos ocupa, las preocupaciones que se privilegian en el contexto educativo y el sistema educativo nacional ocupan el lugar superior y dominante. Una agenda política de este campo implica la insubordinación de dichos saberes sometidos, contra este ordenamiento discursivo, para reconfigurar las relaciones del campo de la didáctica y proponer otras voces en el debate que dibujen nuevas búsquedas.

23 Las condiciones de utilización del discurso que Foucault (1971) propone son el ritual, las sociedades de discurso, las doctrinas y la adecuación social del discurso. 


\section{Bibliografía}

Aprea, Gustavo (2006). Sociedad, información y conocimiento ante la difusión de las TIC. www.igluppiweb. com.ar/tics/obv_soc_info_nota12.htm.

Bernstein, B. (1993). La construcción social del discurso pedagógico. Bogotá: Prodic "El griot”.

Bourdieu, P. (1997). Capital cultural, escuela y espacio social. México: Siglo Veintiuno Editores.

Brünner, José Joaquín. (1999). Globalización cultural y postmodernidad. Chile: Breviarios. Fondo de Cultura Económica.

Callon, M., Courtial, J. P. y Penan, H. (1995). Cienciometría. La medición de la actividad científica: de la bibliometría a la vigilancia tecnológica. Gijón: Trea.

Camilloni,A. (1996). De herencias, deudas y legados. Una introducción a las corrientes actuales en didáctica. En Corrientes didácticas contemporáneas. Argentina: Paidós.

Castañeda Bernal, Elsa (2000). Estudios sociales en educación. Estado del arte, 1990-1999. En Estados del arte de la investigación en educación y pedagogía en Colombia, tomo II. Miriam Henao Willes y Jorge Orlando Castro (comps.). Bogotá: Colciencias-Sociedad Colombiana de Pedagogía.

Cepal y Unesco (1992). Educación y conocimiento: eje de la transformación productiva con equidad.

Cuesta, R. (1997). Clio en las aulas. La enseñanza de la historia en España entre reformas, ilusiones y rutinas. Madrid: Akal.

Departamento de Investigaciones de la Universidad Central. Línea de Investigación en Jóvenes y Culturas Juveniles. Estado del arte del conocimiento producido sobre jóvenes en Colombia, 1985-2003. Programa presidencial Colombia Joven-Agencia de Cooperación alemana GTZ-Unicef Colombia.
Díaz, M. (1993). El campo intelectual de la educación en Colombia. Cali: Textos universitarios, Universidad del Valle.

Foucault, M. (1993). (1 $1^{\text {a }}$ ed., 1966). Las palabras y las cosas. Una arqueología de las ciencias humanas. México: Siglo Veintiuno Editores. (1996). (1a ed., 1969). La arqueología del saber. Barcelona: Siglo Veintiuno Editores. (2002). (1 ${ }^{a}$ ed., 1971). El orden del discurso. Barcelona: Tusquets Editores.

Gvirtz, S. Larripa, S. y Oría, Á. (2004). Prácticas discursivas pedagógicas, didácticas y escolares: algunas categorías para repensar la relación entre el saber y la escuela. Fundación Lúminis. En http://fundacionluminis.org.ar/Biblioteca.html.

Larrosa, J. (1995). Escuela, poder y subjetivación. Madrid: La Piqueta.

Narodowski, Mariano (1999). Después de clase. Desencantos y desafíos de la escuela actual. Buenos Aires: Ediciones Novedades Educativas.

Pérez, A. (2000). La cultura escolar en la sociedad neoliberal. Madrid: Morata.

Sauquillo, J. (1989). Michel Foucault: una filosofía de la acción. Madrid: Centro de Estudios Constitucionales.

Trave González, Gabriel (1998). La investigación en didáctica de las ciencias sociales. Perspectivas y aportaciones desde la enseñanza y el aprendizaje de las nociones económicas. Huelva: Universidad de Huelva.

Unesco: Ibe Education Thesaurus (2002). Revised and enlarged. list of terms for indexing and retrieving documents and data in the field of education. 6th ed. En: http://www.ibe.unesco.org/International/DocServices/Thesaurus/theshome.htm. 